Methods In November 1999 all physical therapists working within the catchment area of two rheumatological centres (Leiden, Enschede) were invited to participate. Education programmes were organised in parallel in both regions and consisted of a 5-day postgraduate training programme, followed by 2-monthly seminars. The programme focussed on examination and treatment of patients with rheumatic diseases and on communication with patients with a chronic disease. Communication among health professionals was enhanced by teaching practices within the rheumatological centres and by developing a draft guideline on communication among health professionals. Outcome measurements included questionnaires on the organisation, didactics and contents of the training programme (6-point scales, range 1 ; very bad to 6 ; very good), knowledge, interdisciplinary communication and patient satisfaction.

Results 63 of the 64 physical therapists (98.4\%) finished the education programme and were formally registered. Evaluation of the training programme measured directly after each part of it showed positive scores with respect to contents, didactics and organisation (all medians 5, ranges 2-6). Knowledge scores increased significantly directly following the training programme ( $p<0.001 ;$ Mann Whitney $U$ ). Measurements regarding the effectiveness on quality of care (interdisciplinary communication and patient satisfaction) will be completed in 2001 .

Conclusions The preliminary results indicate that setting up a system of networks and continuing education for physical therapists with respect to the treatment of patients with rheumatic diseases is feasible. The complete set of results will be available by the end of 2001. Further research will focus on the implementation of the system on a larger scale. Funded by The Health Research and Development Council of The Netherlands; ZON.

\section{SP0170 IMPLEMENTING EDUCATIONAL TRAINING PROGRAMS FOR HEALTH CARE PERSONNEL IN ARTHRITIS}

LX Hansson. Research and Development, The Swedish Rheumatism Association, Stockholm, Sweden

10.1136/annrheumdis-2001.1267

Co-operation for education of health care personal in arthritis.

In Sweden we have 24 County Councils that are responsible for health care.

One of the most important tasks the Swedish Rheumatism Association, the SRA, is to give health care professionals more knowledge so they can discover early arthritis. If they do we today have a good arsenal of therapies to give people with arthritis a hopeful future. The communities themselves don't have resources to give their personnel further education in medical specialties. In Sweden as in other countries the medical companies offer the doctors a lot of specialised education. This is criticised by the public and there are forces in movement to change the situation.

For a long time the Swedish Arthritis/Rheumatism Association has given courses to health care personnel but this has only been the case in some parts of Sweden. Therefore our organisation, with support from 6 medical companies, now has started an educational project. This last autumn we negotiated with the medical companies to support us and in January we employed one person to contact all the County Councils to get a co-operation project with them to plan for educational days i e training for the medical profession.
We have a board, a steering group for this which includes representatives for the organisation of the GPs, for the organisation SRF (the rheumatologists), for Sverefo which represents the professionals from the arthritis team, for our own hospital Spenshult (owned by the SRA) representing the Patient Partner Project in Sweden which is employed by us and some other additional important partners.

The contents of the education is decided upon in cooperation between representatives from the SRA and the county councils mainly on the basis of some educational programs in arthrosis, rheumatoid arthritis, fibromyalgia och osteoporosis which the board picks out. Our patient owned hospital Spenshult in Halmstad on the west coast of Sweden has the main responsibility for the implementation of the educational days and the medical quality. We also try to stress patient influence and the point of view of the patients.

The companies can have exhibitions in the vicinity and treat the participants to food and an attractive free time program. The curriculum usually doesn't contain themes from rheumatology but instead fields like leadership, conflict management and personal development. The educational days vary from a group of 20 participating doctors working with dialogue pedagogy to lectures with 150 participants. We stress a qualitatively good documentation.

The year 2001 is a formative year for the project. Until now we have carried out three educational programs this spring and plan seven for the fall. In 2002, which together with the Swedish Pharmacies has been declared the year of Arthritis/Rheumatism, we plan to carry through approximately forty such educational programs in all county councils in Sweden.

Another project which I want to mention here also covers the education of health care professionals is to build up" e-learning" for the rheuma team.

At our next conference we hope to be able to present some more experiences from both these projects.

\section{Health services, economics and outcome research}

\section{HP0034 RESPONSIVENESS OF SELECTED DISEASE-SPECIFIC PATIENT-ASSESSED INSTRUMENTS IN ANKYLOSING SPONDYLITIS (AS)}

${ }^{1} \mathrm{KL}$ Haywood, ${ }^{2} \mathrm{AM}$ Garratt, ${ }^{3} \mathrm{~K}$ Dziedzic, ${ }^{4} \mathrm{PT}$ Dawes. ${ }^{1}$ Department of Health Sciences and Clinical Evaluation, University of York, York; ${ }^{2}$ Unit of Health Care Epidemiology, Institute of Health Sciences, Oxford; ${ }^{3}$ Primary Care Sciences Research Centre, Keele University, Newcastle-Under-Lyme; ${ }^{4}$ Staffordshire Rheumatology Centre, North Staffordshire Acute Hopsitals Trust, Stoke-on-Trent, UK

\subsection{6/annrheumdis-2001.1268}

Introduction Five instruments, including an individualised measure of AS-related quality of life, the Patient-Generated Index (PGI-AS), have been assessed for measurement properties of reliability, validity and responsiveness in a large group of AS patients. Responsiveness refers to the ability to detect clinically important change and is an essential requirement for evaluative instruments.

Objective To establish comparative evidence in support of instrument longitudinal validity and responsiveness.

Methodology A questionnaire containing all instruments was mailed to over 450 patients randomly selected from six AS databases. Patients completed the questionnaire at baseline (response 